\title{
Meat consumption and the risk of incident distal colon and rectal adenoma
}

\author{
LM Ferrucci' ${ }^{1,2}$, R Sinha', W-Y Huang', SI Berndt', HA Katki', RE Schoen ${ }^{3}$, RB Hayes $^{4}$ and AJ Cross*,I \\ 'Division of Cancer Epidemiology and Genetics, National Cancer Institute, National Institutes of Health, Department of Health and Human Services, \\ Bethesda, MD, USA; ${ }^{2}$ Division of Chronic Disease Epidemiology, Yale School of Public Health, New Haven, CT, USA; ${ }^{3}$ Department of Medicine, University \\ of Pittsburgh, Pittsburgh, PA, USA; ${ }^{4}$ Department of Environmental Medicine, New York University School of Medicine, New York, NY, USA
}

BACKGROUND: Most studies of meat and colorectal adenoma have investigated prevalent events from a single screening, thus limiting our understanding of the role of meat and meat-related exposures in early colorectal carcinogenesis.

METHODS: Among participants in the screening arm of the Prostate, Lung, Colorectal, and Ovarian Cancer Screening Trial who underwent baseline and follow-up sigmoidoscopy $(n=17072)$, we identified 1008 individuals with incident distal colorectal adenoma. We calculated odds ratios (ORs) and 95\% confidence intervals ( $95 \% \mathrm{Cls}$ ) for associations between meat and meat-related components and incident distal colorectal adenoma using multivariate logistic regression.

RESULTS: We observed suggestive positive associations for red meat, processed meat, haeme iron, and nitrate/nitrite with distal colorectal adenoma. Grilled meat $(\mathrm{OR}=1.56,95 \% \mathrm{Cl}=1.04-2.36)$, well or very well-done meat $(\mathrm{OR}=1.59,95 \% \mathrm{Cl}=1.05-2.43)$, 2-amino- I-methyl-6-phenyl-imidazo[4,5-b]pyridine $(\mathrm{PhIP})(\mathrm{OR}=1.75,95 \% \mathrm{Cl}=1.17-2.64)$, benzo[a]pyrene $(\mathrm{OR}=1.53,95 \% \mathrm{Cl}=$ I.06-2.20), and total mutagenic activity $(\mathrm{OR}=1.57,95 \% \mathrm{Cl}=1.03-2.40)$ were positively associated with rectal adenoma. Total iron (diet and supplements) $(\mathrm{OR}=0.69,95 \% \mathrm{Cl}=0.56-0.86)$ and iron from supplements $(\mathrm{OR}=0.65,95 \% \mathrm{Cl}=0.44-0.97)$ were inversely associated with any distal colorectal adenoma.

CONCLUSION: Our findings indicate that several meat-related components may be most relevant to early neoplasia in the rectum. In contrast, total iron and iron from supplements were inversely associated with any distal colorectal adenoma.

British Journal of Cancer (2012) 1 06, 608-616. doi:10.1038/bjc.20 I I.549 www.bjcancer.com

Published online 13 December 2011

(C) 2012 Cancer Research UK

Keywords: colorectal adenoma; diet; meat; haeme iron; meat mutagens; nitrate; nitrite

Consumption of red meat and processed meat has been positively associated with colorectal cancer in numerous epidemiological studies (WCRF/AICR, 2007); however, our understanding of whether these foods have a role during tumour initiation, progression, or both is limited. Few prospective epidemiological studies have been able to assess diet in relation to incident colorectal adenoma, a known precursor to colorectal cancer. This limited research on diet and incident colorectal adenoma has been primarily due to a lack of detailed data on screening procedures for colorectal cancer in prospective cohort studies, as the screenings are typically not conducted as part of these studies. In addition, randomised trials of colorectal adenoma prevention have evaluated recurrence of adenoma rather than incident adenoma. In colorectal adenoma prevention trials, processed meat (Robertson et al, 2005; Martinez et al, 2007), pan-fried meat (Martinez et al, 2007), and well/very well-done meat (Martinez et $a l, 2007)$ have been positively associated with recurrence of advanced or multiple adenoma, whereas dietary iron (Tseng et al, 1997) has been inversely associated with recurrence. However, the impact of diet on the carcinogenic process in individuals with a

*Correspondence: Dr AJ Cross; E-mail: crossa@mail.nih.gov Received 27 September 201 I; revised 16 November 2011; accepted 20 November 201 I; published online 13 December 2011 history of colorectal adenoma may differ from populations without a history of polyps and/or colon disease.

Thus far, most studies of diet, including meat and meat-related exposures, and colorectal adenoma have been for prevalent lesions discovered at a one-time screen (Tseng et al, 1996; Sinha et al, 1999, 2001, 2005c; Chan et al, 2005; Gunter et al, 2005; Shin et al, 2007; Ferrucci et al, 2009; Burnett-Hartman et al, 2011; Fu et al, 2011; Wang et al, 2011). Existing research on meat and incident colorectal adenoma is mixed and comes from five studies within just four different cohorts (Giovannucci et al, 1992; Tranah et al, 2004; Kesse et al, 2006; Wu et al, 2006; Rohrmann et al, 2009).

Several meat-related compounds may each contribute to colorectal neoplasia. Heterocyclic amines (HCAs) and polycyclic aromatic hydrocarbons (PAHs), meat mutagens formed during high-temperature cooking (Sinha et al, 1995, 1998a, b), are gastrointestinal carcinogens in animal models (Ito et al, 1991; Ohgaki et al, 1991; Culp et al, 1998; Ochiai et al, 2002). Meat is also a source of iron, and both non-haeme and haeme iron can induce oxidative DNA damage (Glei et al, 2002; Tappel, 2007). Haeme iron, which is primarily found in red meat and is more bioavailable, has been associated with fecal water cytotoxicity (Sesink et al, 1999, 2000) and promotion of colorectal cancer in rodents (Pierre et al, 2004), as well as endogenous formation of $\mathrm{N}$-nitroso compounds (NOCs) (Cross et al, 2003), powerful multisite carcinogens (Bogovski and Bogovski, 1981; Lijinsky, 1992). 
Additional NOC exposure may occur from intake of nitrate and nitrite, precursors to NOCs that are added to processed meat for both preservation and colour.

With a lack of epidemiological research on the association between meat consumption and meat-related components and incident colorectal adenoma, we investigated this relationship in the screening arm of the Prostate, Lung, Colorectal, and Ovarian (PLCO) Cancer Screening Trial. Repeat flexible sigmoidoscopies conducted as part of the PLCO Cancer Screening Trial provided a unique context for studying adenoma as an incident rather than a prevalent event among a population free of distal colorectal adenoma at baseline, with adenoma status confirmed by follow-up sigmoidoscopy.

\section{MATERIALS AND METHODS}

\section{PLCO Cancer Screening Trial}

The PLCO Cancer Screening Trial is a multi-centre, randomised controlled trial designed to evaluate screening methods for the early detection of prostate, lung, colorectal, and ovarian cancer (Prorok et al, 2000). In brief, 154952 participants (55-74 years of age) were recruited from 10 centres in the United States from 1993 to 2001 and randomly assigned to the screened or non-screened arm. Subjects underwent flexible sigmoidoscopy screening at baseline and then subsequently at either study year 3 or 5 (the protocol was modified in April 1995; changing from a 3- to 5-year interval). Lesions were not removed during trial sigmoidoscopies; subjects with abnormal findings were referred to their health-care providers for diagnostic follow-up. All participants completed a self-administered baseline questionnaire on demographics, personal and family cancer history, medical history, and lifestyle habits. Participants in the screening arm also completed a 137-item food frequency questionnaire (FFQ) on usual intake of foods and beverages during the past year (http://prevention.cancer.gov/files/ programs-resources/dqx.pdf). Overall, $89 \%$ of participants completed the FFQ before or on the same day as the baseline sigmoidoscopy. Institutional Review Boards at the National Cancer Institute and the 10 study centres approved the study and participants provided written informed consent.

\section{Analytic population}

These analyses were restricted to the screening arm of the trial $(n=77445)$. All participants had to have a normal sigmoidoscopy at baseline (i.e., no adenoma, no abnormal/suspicious findings) and not have had colorectal cancer before the baseline screen $(n=49500)$. Individuals with an inadequate $(<50 \mathrm{~cm})$ baseline sigmoidoscopy $(n=7096)$ were considered ineligible. Among individuals who then underwent a PLCO follow-up ( 3 or 5 years after baseline) sigmoidoscopy $(n=29697)$, we excluded those who had colorectal cancer before or at the follow-up screen $(n=16)$ or had an inadequate follow-up sigmoidoscopy $(n=2915)$. Individuals who had a positive finding at follow-up sigmoidoscopy, but at the diagnostic endoscopy no adenoma was found $(n=3370)$; individuals who had a positive finding at follow-up sigmoidoscopy, but a diagnostic endoscopy was not performed $(n=418)$; and persons who had a positive finding at follow-up sigmoidoscopy, but the study was unable to obtain any information regarding diagnostic follow-up $(n=460)$ were also excluded.

For these dietary analyses, we further excluded individuals who lacked either the baseline questionnaire $(n=21)$ or the FFQ $(n=2296)$ and those who missed $>7$ food items or reported energy intake in the top or bottom $1 \%$ on the FFQ $(n=435)$ to remove people with implausible reported energy intake from the self-reported dietary data. Furthermore, we excluded participants who had a self-reported history of Crohn's disease, ulcerative colitis, familial polyposis, Gardner's syndrome, or colorectal polyps $(n=1835)$, or a history of any cancer other than nonmelanoma skin cancer before follow-up sigmoidoscopy $(n=956)$. After these exclusions, our analytic population consisted of 17072 individuals.

\section{Dietary exposures}

Nutrient intake was estimated using the Diet ${ }^{\star}$ Calc Analysis Program (version 1.4.3, 2005, National Cancer Institute, Rockville, MD, USA), which is based on nutrient content information from the United States Department of Agriculture (USDA) Survey Nutrient Database and the Nutrition Data Systems for Research from the University of Minnesota. Intake ( $g$ per day) was calculated from frequency and portion size information. Red meat included beef, pork, and lamb products, whereas white meat encompassed poultry and fish. Processed meat included ham, hot dogs, liver, cold cuts, sausage, and bacon.

Questions on meat-cooking methods (barbequing, grilling, pan frying, and broiling) and doneness level (rare, medium, well, or very well done) for steak, bacon, sausage, pork chops, and hamburgers were included in the FFQ. With the Computerized Heterocyclic Amines Resource for Research in Epidemiology of Disease (CHARRED) (http://www.charred.cancer.gov) software application, we generated intake estimates of three HCAs (ng per day): 2-amino-3,4,8-trimethylimidazo[4,5-f]quinoxaline (DiMeIQx), 2-amino-3,8-dimethylimidazo[4,5-f]quinoxaline (MeIQx), and 2-amino-1-methyl-6-phenyl-imidazo[4,5-b]pyridine (PhIP), as well as benzo $[a]$ pyrene $(\mathrm{B}[a] \mathrm{P})$, a marker of PAH exposure (Sinha et al, 2005a). Total mutagenic activity in meat (revertant colonies per day) determined by the standard plate incorporation assay with Salmonella typhimurium strain TA98 (Ames et al, 1975) was also estimated.

Meat-cooking data were used to estimate haeme iron intake from meat using a haeme iron database based on measured values from meat samples (Sinha et al, 2005a). Total iron (non-haeme and haeme) from meat (limited to those meats in the haeme iron database) was estimated from the nutrient content information in the USDA Survey Nutrient Database. Nitrate and nitrite intake from processed meats was calculated using a database of laboratory measured values of these compounds in 10 types of processed meats representing $90 \%$ of processed meats assessed by a typical FFQ in the United States (Sinha et al, 2005a).

\section{Outcome ascertainment}

Incident distal colorectal adenoma cases were individuals with a positive follow-up sigmoidoscopy who then had a diagnostic follow-up endoscopy (e.g., colonoscopy, flexible sigmoidoscopy) conducted outside the study during which a distal colorectal adenoma diagnosis was confirmed. The PLCO personnel abstracted follow-up endoscopy records using a standard form that captured polyp histology, size, and location. Adenoma cases were restricted to those in the distal colon or rectum, as individuals with no abnormal/suspicious findings at follow-up sigmoidoscopy did not undergo diagnostic endoscopy. Cases were further classified into multiple distal colorectal adenoma (two or more adenoma) and advanced adenoma (size of $\geqslant 1 \mathrm{~cm}$, high-grade dysplasia, or villous components, including tubulovillous); with some individuals falling into both categories.

\section{Statistical analysis}

Quartile cut points for dietary variables were based on intake of the analytic cohort. Odds ratios (OR) and $95 \%$ confidence intervals (95\% CIs) were computed using unconditional logistic regression, with the first quartile as the referent group. Meat intake was nutrient density adjusted; using residual energy adjustment did 
not alter our findings (Willett, 1998). Total iron intake from diet and supplements was calculated by residually energy adjusting dietary iron and adding it to supplemental iron (mg per day). Multivariate models were adjusted for the following characteristics: age at baseline, study centre, gender, ethnicity, education, family history of colorectal cancer, body mass index, use of non-steroidal anti-inflammatory drugs, physical activity, smoking status, and intakes of alcohol, dietary calcium, supplemental calcium, dietary fibre, and total energy. To test for heterogeneity between the anatomical subsites (distal colon vs rectum), we calculated the weighted average of the two $\beta$-coefficients from the logistic regression model, with weights being proportional to the inverse of the variances. We then calculated the following $\chi^{2}$ statistic with one degree of freedom: $T=\sum_{i=1}^{2}\left(\hat{\beta}_{i}-\bar{\beta}\right)^{2} / \sigma_{i}^{2}$, where $\hat{\beta}_{i}$ and $\sigma_{i}^{2}$ are the coefficient and its variance for each subsite, and $\bar{\beta}$ is the weighted average of the $\beta$-coefficients. We also evaluated potential variation in risk estimates by gender, screening interval (3 or 5 years), and multiple and/or advanced adenoma. Trend tests were calculated using the median intake values of each quartile. $P$-values are two sided and analyses were conducted using SAS software (SAS Institute, Cary, NC, USA, version 9.2).

\section{RESULTS}

We identified 1008 individuals with incident distal colorectal adenoma 3-5 years after baseline, leaving 16064 individuals with no abnormal/suspicious findings at both baseline and follow-up flexible sigmoidoscopies. Of the 1008 individuals with distal colorectal adenoma, 503 cases were non-advanced, 237 were advanced, and 268 were unknown for advanced status. A total of 131 individuals had multiple adenomas at follow-up and 772 had at least one adenoma in the distal colon, whereas 263 had at least one adenoma in the rectum (the specific location within the distal colorectum was unknown for 25 individuals). Individuals in the highest quartile of red meat were more likely to be non-Hispanic White and be current smokers compared with those in the lowest quartile of red meat consumption (Table 1). Participants who consumed the most red meat were also less likely to be female, less educated, and less physically active than those who consumed the least red meat.

We observed borderline statistically significant positive associations in the top quartiles of red (quartile $4 v s$ quartile $1 \mathrm{OR}=1.22$, $95 \% \mathrm{CI}=0.98-1.52 ; P$-trend $=0.12)$ and processed $(\mathrm{OR}=1.23$, $95 \% \mathrm{CI}=0.99-1.54 ; P$-trend $=0.12)$ meat in relation to incident distal colorectal adenoma (Table 2). There was no heterogeneity between the risks observed for distal colon adenoma $(\mathrm{OR}=1.22$, $95 \% \mathrm{CI}=0.95-1.56)$ and rectal adenoma $(\mathrm{OR}=1.33,95 \%$ $\mathrm{CI}=0.87-2.04)$ for red meat intake $\left(P_{\text {heterogeneity }}=0.73\right)$. In contrast, the risk estimate observed for processed meat and colon adenoma $(\mathrm{OR}=1.24,95 \% \mathrm{CI}=0.97-1.59)$ was higher than the risk estimate for rectal adenoma $(\mathrm{OR}=1.08,95 \% \mathrm{CI}=0.71-1.65)$, although this difference was not statistically significant $\left(P_{\text {heterogeneity }}=0.58\right)$. Risk estimates for advanced and/or multiple adenoma (323 cases) were not statistically significant for red $(\mathrm{OR}=1.32,95 \% \mathrm{CI}=0.91-1.94)$ or processed meat $(\mathrm{OR}=1.12$, $95 \% \mathrm{CI}=0.76-1.65)$. White meat intake was not associated with incident distal colorectal adenoma; there was also no clear association when separated into chicken (quartile $4 v s$ quartile 1 $\mathrm{OR}=0.95,95 \% \quad \mathrm{CI}=0.78-1.15) \quad$ and fish $\quad(\mathrm{OR}=1.16,95 \%$ $\mathrm{CI}=0.96-1.40)$.

There were some suggestive positive associations for grilled meat $(\mathrm{OR}=1.20,95 \% \mathrm{CI}=0.97-1.49)$, meat cooked well or very well-done $(\mathrm{OR}=1.20,95 \% \mathrm{CI}=0.95-1.49)$, and $\mathrm{PhIP}(\mathrm{OR}=1.18$, $95 \% \mathrm{CI}=0.96-1.45)$ and colorectal adenoma for individuals in the top quartiles of intake compared with those in the lowest quartiles (Table 3). Associations for grilled meat $(\mathrm{OR}=1.56,95 \% \mathrm{CI}=$ $1.04-2.36$ vs $\left.\mathrm{OR}=1.18,95 \% \mathrm{CI}=0.93-1.51 ; P_{\text {heterogeneity }}=0.26\right)$, well or very well-done meat $(\mathrm{OR}=1.59,95 \% \mathrm{CI}=1.05-2.43$ vs

Table I Means and proportions ${ }^{\mathrm{a}}$ of baseline characteristics by red meat quartile $(N=17072)$

\begin{tabular}{|c|c|c|c|c|}
\hline Characteristics & \multicolumn{4}{|c|}{ Quartile of red meat intake } \\
\hline $\begin{array}{l}\text { Red meat (g per } 1000 \mathrm{kcal}) \text {, mean } \pm \text { s.d. } \\
\text { Age (years), mean } \pm \text { s.d. } \\
\text { Female, } n(\%)\end{array}$ & $\begin{array}{r}12.8 \pm 4.9 \\
62.8 \pm 5.3 \\
2589(60.7)\end{array}$ & $\begin{array}{r}26.0 \pm 3.5 \\
62.5 \pm 5.2 \\
2190(51.3)\end{array}$ & $\begin{array}{r}39.0 \pm 4.3 \\
62.2 \pm 5.1 \\
1710(40.1)\end{array}$ & $\begin{array}{l}65.7 \pm 18.8 \\
61.5 \pm 4.9 \\
1130(26.5)\end{array}$ \\
\hline $\begin{array}{l}\text { Education, college graduate or postgraduate, } n(\%) \\
\text { Body mass index }\left(\mathrm{kg} \mathrm{m}^{-2}\right) \text {, mean } \pm \text { s.d. } \\
\text { Physical activity, } 4+\text { hours per week, } n(\%)\end{array}$ & $\begin{array}{r}1993(46.7) \\
25.7 \pm 4.3 \\
|45|(34.0)\end{array}$ & $\begin{array}{r}1799(42.2) \\
26.7 \pm 4.4 \\
1113(26.1)\end{array}$ & $\begin{array}{r}1572(36.8) \\
27.6 \pm 4.5 \\
1005(23.6)\end{array}$ & $\begin{array}{r}1333(31.2) \\
28.5 \pm 4.7 \\
842(19.7)\end{array}$ \\
\hline $\begin{array}{l}\text { Alcohol (g per day), mean } \pm \text { s.d. } \\
\text { Family history of colorectal cancer, } n(\%) \\
\text { Non-steroidal anti-inflammatory drugs, } 60+\text { pills per month, } n \text { (\%) } \\
\text { Total energy intake (kcal per day), mean } \pm \text { s.d. } \\
\text { White meat (g per } 1000 \mathrm{kcal}), \text { mean } \pm \text { s.d. } \\
\text { Dietary calcium (mg per } 1000 \mathrm{kcal}) \text {, mean } \pm \text { s.d. } \\
\text { Supplemental calcium (mg per day), mean } \pm \text { s.d. } \\
\text { Fibre (g per } 1000 \mathrm{kcal}), \text { mean } \pm \text { s.d. }\end{array}$ & $\begin{array}{l}9.0 \pm 25.4 \\
371(8.7) \\
459(10.8) \\
1934 \pm 705 \\
28.8 \pm 23.6 \\
530 \pm 194 \\
359 \pm 414 \\
14.1 \pm 4.1\end{array}$ & $\begin{array}{l}10.3 \pm 22.1 \\
355(8.3) \\
499(11.7) \\
2001 \pm 758 \\
26.4 \pm 19.1 \\
488 \pm 160 \\
309 \pm 395 \\
12.3 \pm 3.3\end{array}$ & $\begin{array}{l}11.4 \pm 22.4 \\
356(8.3) \\
506(11.9) \\
2122 \pm 796 \\
25.5 \pm 17.8 \\
458 \pm 145 \\
245 \pm 351 \\
11.2 \pm 2.9\end{array}$ & $\begin{array}{l}11.0 \pm 19.6 \\
389(9.1) \\
496(11.6) \\
2277 \pm 882 \\
25.4 \pm 17.4 \\
413 \pm 127 \\
185 \pm 304 \\
10.0 \pm 2.7\end{array}$ \\
\hline
\end{tabular}

May not sum to $100 \%$ because of missing data or rounding. 
Table 2 Multivariate ORs and 95\% Cls for meat intake (g per $1000 \mathrm{kcal}$ ) and incident distal colorectal adenoma

\begin{tabular}{|c|c|c|c|c|c|c|}
\hline \multirow[b]{2}{*}{ Characteristics } & \multicolumn{2}{|c|}{ Any distal adenoma $(n=1008)$} & \multicolumn{2}{|c|}{ Descending/sigmoid colon adenoma $(n=772)$} & \multicolumn{2}{|c|}{ Rectal adenoma $(n=263)$} \\
\hline & Cases & $O R^{a}(95 \% \mathrm{Cl})$ & Cases & $O R^{a}(95 \% \mathrm{Cl})$ & Cases & $O R^{a}(95 \% \mathrm{Cl})$ \\
\hline$Q \mid(\mid 3.5)$ & 185 & 1.00 & 144 & 1.00 & 46 & 1.00 \\
\hline Q2 (26.0) & 243 & $1.18(0.96-1.45)$ & 179 & $1.12(0.89-1.41)$ & 66 & $1.30(0.88-1.92)$ \\
\hline Q3 (38.7) & 266 & $1.16(0.94-1.44)$ & 207 & $1.17(0.92-1.48)$ & 67 & $1.19(0.79-1.79)$ \\
\hline Q4 (60.1) & 314 & $1.22(0.98-1.52)$ & 242 & $1.22(0.95-1.56)$ & 84 & $1.33(0.87-2.04)$ \\
\hline \multicolumn{7}{|c|}{ White meat (median) } \\
\hline Q। (9.3) & 235 & 1.00 & 193 & 1.00 & 53 & 1.00 \\
\hline Q2 (17.2) & 265 & $1.65(0.96-1.39)$ & 200 & $1.06(0.86-1.30)$ & 73 & $1.37(0.96-1.96)$ \\
\hline Q3 (26.8) & 274 & $1.22(1.02-1.48)$ & 198 & $1.07(0.87-1.32)$ & 76 & $1.46(1.01-2.09)$ \\
\hline Q4 (45.8) & 234 & $1.08(0.88-|.3|)$ & 181 & $1.01(0.8 \mid-1.25)$ & 61 & $1.19(0.81-1.74)$ \\
\hline$P$-trend ${ }^{b}$ & & 0.60 & & 0.90 & & 0.36 \\
\hline \multicolumn{7}{|c|}{ Processed meat ${ }^{c}$ (median) } \\
\hline Q4 (I5.5) & 324 & $1.23(0.99-1.54)$ & 252 & $1.24(0.97-1.59)$ & 77 & $1.08(0.7 \mid-1.65)$ \\
\hline$P$-trend ${ }^{b}$ & & 0.12 & & 0.14 & & 0.92 \\
\hline
\end{tabular}

Abbreviations: $\mathrm{BMI}=$ body mass index; $\mathrm{Cl}=$ confidence interval; $\mathrm{NSAID}=$ non-steroidal anti-inflammatory drug; $\mathrm{OR}=$ odds ratio. ${ }^{\mathrm{a}} \mathrm{OR}$ adjusted for age at baseline $(\leqslant 59$, 60-64, 65-70, 70+ years), study centre (10 centres), gender, ethnicity (non-Hispanic White, non-Hispanic Black, Hispanic, other), education ( $\leqslant$ high school, some college or post-high school training, college graduate, or postgraduate), family history of colorectal cancer (yes, no, missing), BMl ( $<25,25-<30, \geqslant 30 \mathrm{~kg} \mathrm{~m}^{-2}$, missing), NSAIDs use $(<4,4-29,30-59,60+$ pills per month), physical activity $(0,<1,2,3,4+$ hours per week), smoking status (never, former, current), alcohol intake (g per day, continuous), dietary calcium (mg per $1000 \mathrm{kcal}$, continuous), supplemental calcium (mg per day, continuous), dietary fibre (g per $1000 \mathrm{kcal}$, continuous), and total energy intake (kcal per day, continuous). ${ }^{b}$-trend calculated using the median of each quartile. ${ }^{C}$ Includes bacon, sausage, hot dogs, ham, liver, and cold cuts.

$\left.\mathrm{OR}=1.14,95 \% \mathrm{CI}=0.89-1.46 ; P_{\text {heterogeneity }}=0.18\right)$, and $\mathrm{PhIP}$ intake $(\mathrm{OR}=1.75,95 \% \mathrm{CI}=1.17-2.64$ vs $\mathrm{OR}=1.07,95 \% \mathrm{CI}=$ $\left.0.85-1.36 ; P_{\text {heterogeneity }}=0.04\right)$ seemed stronger for rectal adenoma than colon adenoma. Although there were no clear associations for mutagenic activity and $\mathrm{B}[\mathrm{a}] \mathrm{P}$ intake in relation to any distal colorectal adenoma, both exposures were positively associated with rectal adenoma $(\mathrm{OR}=1.57,95 \% \mathrm{CI}=1.03-2.40 ; \mathrm{OR}=1.53$, $95 \% \mathrm{CI}=1.06-2.20$, respectively), but not colon adenoma $(\mathrm{OR}=0.96,95 \% \mathrm{CI}=0.76-1.22 ; \mathrm{OR}=0.92,95 \% \mathrm{CI}=0.74-1.15$, respectively) with evidence of heterogeneity by subsite $\left(P_{\text {heterogeneity }}=0.05 ; P_{\text {heterogeneity }}=0.02\right.$ for mutagenic activity and $\mathrm{B}[a] \mathrm{P}$, respectively). Analyses of advanced and/or multiple adenoma also revealed a positive association for well or very well-done meat $(\mathrm{OR}=1.45,95 \% \mathrm{CI}=1.01-2.09)$, but there was no variation by adenoma type for the other cooking variables or meat mutagens (data not shown).

Total iron intake was inversely associated with colorectal adenoma, as those who had the highest intake were $31 \%$ less likely to have an adenoma than were those with the lowest intake $(\mathrm{OR}=0.69,95 \% \mathrm{CI}=0.56-0.86, P$-trend $=0.01)$ (Table 4$)$. Analysing dietary and supplementary iron separately revealed an inverse association for iron from supplements $(\mathrm{OR}=0.65,95 \% \mathrm{CI}=0.44-$ $0.97, P$-trend $=0.02$ ), and a similar, although not statistically significant, inverse association for dietary iron $(\mathrm{OR}=0.85,95 \%$ $\mathrm{CI}=0.68-1.07, P$-trend $=0.06)$. In contrast, there was a borderline positive association between haeme iron from meat and colorectal adenoma in the top quartile of intake $(\mathrm{OR}=1.23,95 \% \mathrm{CI}=0.99$ $1.52)$, with evidence for a linear relationship $(P$-trend $=0.03)$. We also observed a suggestive positive association for the highest intake of nitrite plus nitrate from processed meat compared with lowest $(\mathrm{OR}=1.22,95 \% \mathrm{CI}=0.94-1.53)$, although the linear trend was not statistically significant $(P$-trend $=0.14)$. Associations for iron exposures and nitrate plus nitrite did not vary when we investigated advanced and/or multiple adenoma (data not shown) and there was little evidence for differences by anatomical subsite.
Interactions by gender were not statistically significant $(P>0.05)$ (data not shown). In addition, we evaluated all associations stratified by time between baseline and follow-up flexible sigmoidoscopy screening ( 3 or 5 years) and risk estimates were not substantially different (data not shown).

\section{DISCUSSION}

In this comprehensive investigation of meat and meat-related compounds in relation to incident distal colorectal adenoma, we observed borderline positive associations for red meat, processed meat, grilled meat, well or very well-done meat, PhIP, haeme iron from meat, as well as nitrate and nitrite from processed meat. However, in analyses by anatomical subsite, there were statistically significant positive associations for rectal adenoma with grilled meat, well or very well-done meat, PhIP, $\mathrm{B}[a] \mathrm{P}$, and total mutagenic activity. We also found statistically significant inverse associations between total iron (diet and supplements) and iron from supplements and distal colorectal adenoma.

Although epidemiological evidence indicates red meat consumption is a risk factor for colorectal cancer (WCRF/AICR, 2007), studies of colorectal adenoma are not as clear with some positive associations for prevalent adenoma (risk estimates ranging from to 1.4 to 2.02 for highest $v s$ lowest) (Sinha et al, 1999; Ferrucci et al, 2009; Fu et al, 2011), but also several null findings for both prevalent (Gunter et al, 2005; Sinha et al, 2005c; Shin et al, 2007; Burnett-Hartman et al, 2011; Wang et al, 2011) and recurrent adenoma (Mathew et al, 2004; Robertson et al, 2005; Martinez et al, 2007), with risk estimates ranging from 0.88 to 1.19 . Studies of meat and incident colorectal adenoma are similarly inconclusive. There was a positive association in men between the ratio of red meat to white meat and incident adenoma of the distal colon and rectum $(\mathrm{RR}=1.83,95 \% \mathrm{CI}=1.12-3.00)$ (Giovannucci et al, 1992); however, with the accrual of additional cases, an analysis of adenoma of the distal colon only found positive associations for 
Table 3 Multivariate ORs and 95\% Cls for meat-cooking methods (g per $1000 \mathrm{kcal}$ ), doneness levels (g per $1000 \mathrm{kcal}$ ), individual meat mutagens (ng per day), and total mutagenic activity (revertant colonies per day) in relation to incident distal colorectal adenoma

\begin{tabular}{|c|c|c|c|c|c|c|}
\hline Characteristics & \multicolumn{2}{|c|}{ Any distal adenoma $(n=1008)$} & \multicolumn{2}{|c|}{ Descending/sigmoid colon adenoma $(n=772)$} & \multicolumn{2}{|c|}{ Rectal adenoma $(n=263)$} \\
\hline QI (0.0) & 225 & 1.00 & 176 & 1.00 & 55 & 1.00 \\
\hline Q2 (1.5) & 212 & $1.12(0.91-1.38)$ & 163 & $1.10(0.88-1.40)$ & 54 & $1.20(0.80-1.79)$ \\
\hline Q3 (6.1) & 271 & $1.26(1.03-1.54)$ & 202 & $1.21(0.96-1.52)$ & 68 & $1.36(0.92-2.03)$ \\
\hline Q4 (16.4) & 300 & $1.20(0.97-1.49)$ & 231 & $1.18(0.93-1.51)$ & 86 & $1.56(1.04-2.36)$ \\
\hline \multicolumn{7}{|c|}{ Pan fried meat (median) } \\
\hline QI (0.3) & 209 & 1.00 & 157 & 1.00 & 58 & 1.00 \\
\hline Q2 (1.3) & 255 & $1.06(0.87-1.29)$ & 194 & $1.08(0.86-1.34)$ & 67 & $0.99(0.68-1.43)$ \\
\hline Q3 (3.8) & 269 & $1.05(0.86-1.28)$ & 208 & $1.08(0.86-1.35)$ & 67 & $0.94(0.64-1.37)$ \\
\hline Q4 ( 11.7$)$ & 275 & $1.03(0.83-1.28)$ & 213 & $1.04(0.81-1.34)$ & 71 & $1.02(0.68-1.54)$ \\
\hline P-trend ${ }^{\mathrm{b}}$ & & 0.80 & & 0.76 & & 0.99 \\
\hline P-trend ${ }^{b}$ & & 0.67 & & 0.79 & & 0.14 \\
\hline \multicolumn{7}{|l|}{ DiMelQx } \\
\hline QI (0.0) & 233 & 1.00 & 189 & 1.00 & 54 & 1.00 \\
\hline Q2 (0.5) & 244 & $1.02(0.84-1.23)$ & 188 & $0.96(0.78-1.18)$ & 59 & $1.07(0.74-1.56)$ \\
\hline Q3 (1.4) & 241 & $0.95(0.78-1.14)$ & 182 & $0.87(0.71-1.08)$ & 69 & $1.19(0.83-1.72)$ \\
\hline Q4 (3.8) & 288 & $0.99(0.82-1.20)$ & 213 & $0.89(0.72-1.11)$ & 79 & $1.20(0.82-1.74)$ \\
\hline$P$-trend ${ }^{b}$ & & 0.85 & & 0.34 & & 0.38 \\
\hline \multicolumn{7}{|l|}{ MelQx } \\
\hline QI (4.8) & 202 & 1.00 & 162 & 1.00 & 48 & 1.00 \\
\hline Q2 (13.1) & 255 & $1.12(0.92-1.36)$ & 189 & $1.04(0.83-1.29)$ & 66 & $1.22(0.83-1.79)$ \\
\hline Q3 (26.4) & 262 & $1.04(0.85-1.27)$ & 196 & $0.97(0.77-1.22)$ & 71 & $1.19(0.81-1.77)$ \\
\hline \multicolumn{7}{|l|}{$B[a] P$} \\
\hline Q। (0.5) & 225 & 1.00 & 184 & 1.00 & 50 & 1.00 \\
\hline Q2 (2.7) & 253 & $1.01(0.83-1.22)$ & 193 & $0.93(0.75-1.15)$ & 64 & $1.15(0.79-1.69)$ \\
\hline Q3 (17.3) & 244 & $1.03(0.85-1.24)$ & 193 & $0.99(0.80-1.22)$ & 57 & $1.09(0.74-1.60)$ \\
\hline Q4 (79.0) & 284 & $1.06(0.88-1.29)$ & 202 & $0.92(0.74-1.15)$ & 90 & $1.53(1.06-2.20)$ \\
\hline P-trend ${ }^{b}$ & & 0.46 & & 0.60 & & 0.02 \\
\hline \multicolumn{7}{|l|}{ Mutagenic activity } \\
\hline QI (692) & 200 & 1.00 & 163 & 1.00 & 42 & 1.00 \\
\hline Q2 (2146) & 260 & $1.13(0.93-1.37)$ & 192 & $1.01(0.81-1.26)$ & 74 & $1.58(1.07-2.34)$ \\
\hline Q3 (43/2) & 249 & $0.97(0.79-1.19)$ & 195 & $0.92(0.73-1.15)$ & 58 & $1.13(0.74-1.73)$ \\
\hline Q4 (9902) & 297 & $1.06(0.86-1.31)$ & 222 & $0.96(0.76-1.22)$ & 87 & $1.57(1.03-2.40)$ \\
\hline$P$-trend ${ }^{b}$ & & 0.46 & & 0.73 & & 0.15 \\
\hline
\end{tabular}

Abbreviations: $\quad \mathrm{BMI}=$ body mass index; $\mathrm{Cl}=$ confidence interval; $\quad$ DiMelQx=2-amino-3,4,8-trimethylimidazo[4,5-f quinoxaline; MelQx=2-amino-3,8-dimethylimidazo [4,5-f]quinoxaline; NSAID = non-steroidal anti-inflammatory drug; OR = odds ratio; PhIP = 2-amino-I-methyl-6-phenyl-imidazo[4,5-b]pyridine. ${ }^{\mathrm{a}} \mathrm{OR}$ adjusted for age at baseline $(\leqslant 59,60-64,65-70,70+$ years), study centre (10 centres), gender, ethnicity (non-Hispanic White, non-Hispanic Black, Hispanic, other), education ( $\leqslant$ high school, some college or post-high school training, college graduate, or postgraduate), family history of colorectal cancer (yes, no, missing), BMI ( $<25,25-<30, \geqslant 30 \mathrm{~kg} \mathrm{~m}{ }^{-2}$, missing), NSAIDs use ( $<4,4-29,30-59,60+$ pills per month), physical activity $(0,<1,2,3,4+$ hours per week), smoking status (never, former, current), alcohol intake (g per day, continuous), dietary calcium ( $\mathrm{mg}$ per $1000 \mathrm{kcal}$, continuous), supplemental calcium (mg per day, continuous), dietary fibre (g per 1000 kcal, continuous), and total energy intake (kcal per day, continuous). ${ }^{b}$-trend calculated using the median of each quartile.

processed meat $(\mathrm{RR}=1.52,95 \% \mathrm{CI}=1.12-2.08)$, but no association with red meat $(\mathrm{RR}=1.18,95 \% \mathrm{CI}=0.87-1.62)(\mathrm{Wu}$ et al, 2006). Among women, a Western dietary pattern high in meat was associated with incident distal colorectal adenoma in a French cohort $(\mathrm{RR}=1.39,95 \% \mathrm{CI}=1.00-1.83)$ (Kesse et al, 2006). Red and processed meat combined were also associated with colon, but not rectal, adenoma in a sample of both men and women $(\mathrm{RR}=1.63,95 \% \mathrm{CI}=1.01-2.30)$ (Rohrmann et al, 2009). Finally, 
Table 4 Multivariate ORs and 95\% Cls for total iron (mg per day), dietary iron (mg per $1000 \mathrm{kcal}$ ), iron and haeme iron from meat (mg per I000 kcal), iron from supplements (mg per day), and nitrate plus nitrite (mg per $1000 \mathrm{kcal}$ ) and incident distal colorectal adenoma

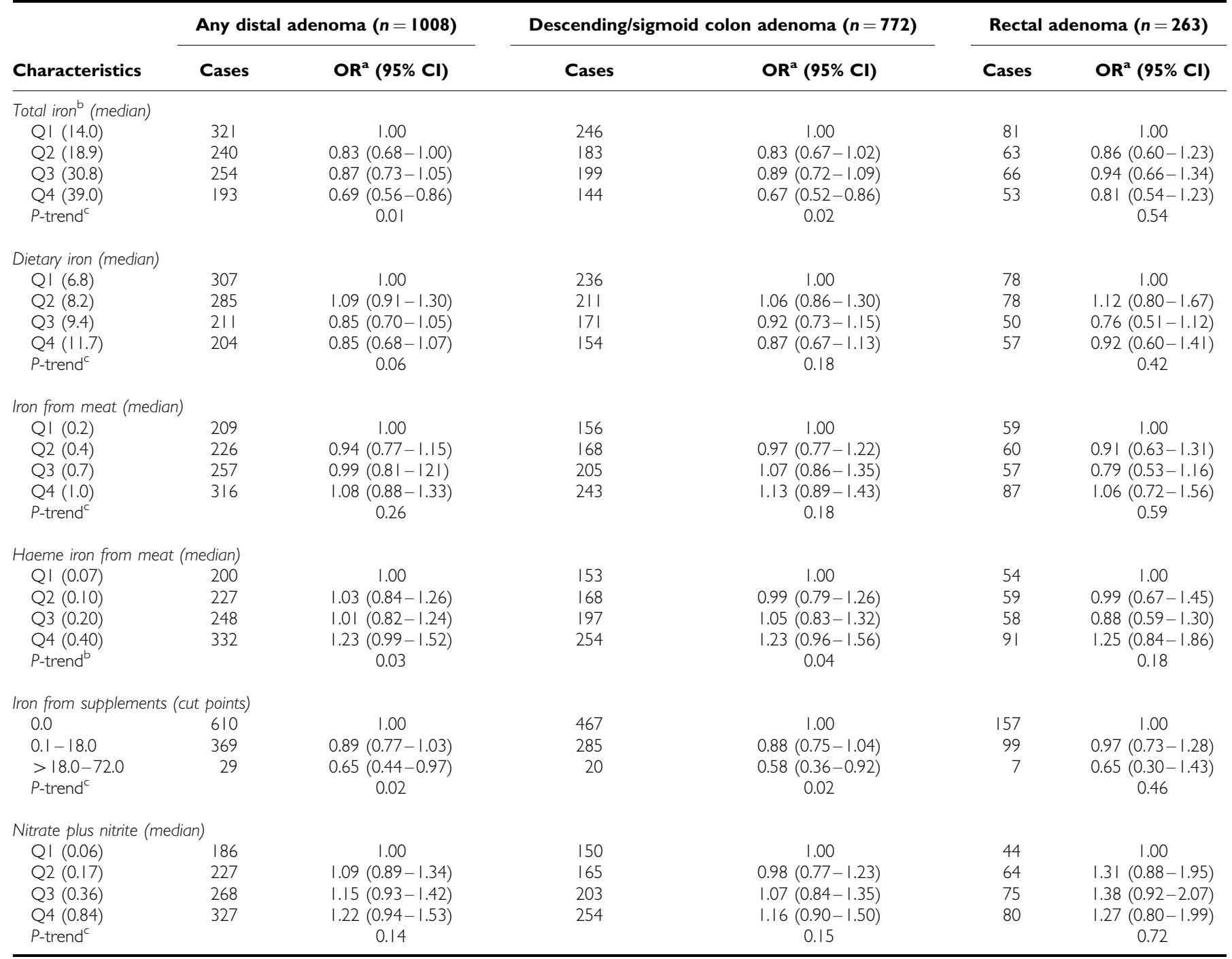

Abbreviations: $\mathrm{BMI}=$ body mass index; $\mathrm{Cl}=$ confidence interval; $\mathrm{NSAID}=$ non-steroidal anti-inflammatory drug; $\mathrm{OR}=$ odds ratio. ${ }^{\mathrm{a}} \mathrm{OR}$ adjusted for age at baseline $(\leqslant 59$, 60-64, 65-70, 70+ years), study centre (10 centres), gender, ethnicity (non-Hispanic White, non-Hispanic Black, Hispanic, other), education ( $\leqslant$ high school, some college or post-high school training, college graduate, or postgraduate), family history of colorectal cancer (yes, no, missing), BMI ( $<25,25-<30, \geqslant 30 \mathrm{~kg} \mathrm{~m}{ }^{-2}$, missing), NSAIDs use $(<4,4-29,30-59,60+$ pills per month), physical activity $(0,<1,2,3,4+$ hours per week), smoking status (never, former, current), alcohol intake (g per day, continuous), dietary calcium (mg per $1000 \mathrm{kcal}$, continuous), supplemental calcium (mg per day, continuous), dietary fibre ( $\mathrm{g}$ per $1000 \mathrm{kcal}$, continuous), and total energy intake (kcal per day, continuous). ${ }^{b}$ Dietary iron (residual energy adjusted) plus iron from supplements. Nutrients in this model were adjusted using residual energy adjustment. ${ }^{\mathrm{C}}$-trend calculated using the median of each quartile or category.

in a subset of the incident distal colorectal adenoma cases $(n=356)$ and controls $(n=396)$ from the PLCO Cancer Screening Trial, red meat was associated with a $59 \%$ statistically significant increased risk of disease (Cross et al, 2011), but this was not replicated in the full sample analysed here. As other populations have not had access to colorectal screening results for all participants, instead relying on self-report of negative adenoma status as screenings were not a part of the study, these previous studies may suffer from false-negative reporting among controls. Similarly, adenoma-free status at baseline for both cases and controls in previous analyses was determined solely by self-report. These studies have been further limited by a low number of cases $(<600)$ reducing the power to detect modest associations.

Although there were only suggestive associations for meatcooking methods, doneness level, and meat mutagens in relation to any distal colorectal adenoma in our population, we observed a statistically significant elevated risk of rectal adenoma with high levels of grilled meat, well or very well-done meat, $\mathrm{PhIP}, \mathrm{B}[a] \mathrm{P}$, and total mutagenic activity. Several studies have observed positive associations between intake of well-done meats or meats cooked by high-temperature methods and colorectal cancer (Gerhardsson de Verdier et al, 1991; Sinha and Rothman, 1999; Le Marchand et al, 2002; Butler et al, 2003; Murtaugh et al, 2004) and prevalent (Sinha et al, 1999, 2005c; Gunter et al, 2005; Fu et al, 2011) or recurrent (Martinez et al, 2007) colorectal adenoma. There is limited and inconclusive evidence on meat mutagens in relation to colorectal cancer, as some studies have found positive associations with colorectal cancer (risk estimates ranging from 1.16 to 4.09 ) (Kampman et al, 1999; Nowell et al, 2002; Butler et al, 2003; Cross et al, 2010), yet others have been null (Murtaugh et al, 2004) or inverse (Augustsson et al, 1999), with risk estimates ranging from 0.60 to 0.89 . Results from prevalent and recurrent adenoma studies point towards a potential role for HCAs (Sinha et al, 2001, 
2005c; Martinez et al, 2007; Ferrucci et al, 2009; Fu et al, 2011; Wang et al, 2011), total mutagenic activity of meat (Sinha et al, 2001; Fu et al, 2011), and B[a]P (Gunter et al, 2005; Sinha et al, 2005b, 2005c; Fu et al, 2011) in early neoplastic changes. With regard to incident adenoma, there was no association in women $(\mathrm{RR}=1.01,95 \% \mathrm{CI}=0.74-1.39)$ or men $(\mathrm{RR}=1.13,95 \% \mathrm{CI}=$ 0.83-1.53) for charred meat (Tranah et al, 2004), suggestive positive associations with MeIQx $(\mathrm{RR}=1.28,95 \% \mathrm{CI}=0.95-1.71)$ and mutagenic activity $(\mathrm{RR}=1.29,95 \% \mathrm{CI}=0.97-1.72)$ in men (Wu et al, 2006), and positive associations in a mixed gender sample for PhIP and browned meat, with increased risks of 36 and $47 \%$, respectively (Rohrmann et al, 2009).

As red meat was more strongly associated with rectal cancers as compared with colon cancers in a recent large prospective cohort (Cross et al, 2010), some of the mixed findings for colorectal adenoma may be due, in part, to the composition of cases by anatomical subsite. We observed a similar suggestive pattern of stronger associations for meat cooking-related exposures in relation to rectal adenoma. Although there is biological plausibility for variation in aetiology by anatomical site in the colorectum, not all studies have observed clear variation in meat-related risks by subsite for prevalent ( $\mathrm{Fu}$ et al, 2011) or incident (Rohrmann et al, 2009) adenoma. As more studies evaluate meat and colorectal neoplasia, it will be important to further evaluate whether associations differ by location. Differences in risk for the various colorectal neoplasia end points could also be present if meat and potentially carcinogenic meat-related exposures are more relevant in colorectal tumour initiation or progression, or if these exposures affect risk differently in individuals in whom neoplastic changes have already occurred in the colorectum.

Thus far, haeme iron intake from meat estimated using a database of measured values has only been evaluated in two studies of colorectal adenoma, in which there were positive, but non-significant associations (risk estimates from 1.46 to 1.50) (Ferrucci et al, 2009; Cross et al, 2011), likely because of limited power, and in the NIH-AARP Diet and Health Study for colorectal cancer, in which there was a suggestive positive association with colorectal cancer $(\mathrm{HR}=1.13,95 \% \mathrm{CI}=0.99-1.29, P$-trend $=0.022)$ (Cross et al, 2010). Other studies of haeme iron and colorectal neoplasia have used rough approximations of haeme iron from meat (Lee et al, 2004; Chan et al, 2005; Larsson et al, 2005; Balder et al, 2006; Kabat et al, 2007), and therefore may not be comparable to results using a quantitative database. Of note, as the haeme iron database does not contain all meats, our risk estimates likely underestimate true associations in this population. As sources of non-haeme iron are generally healthy (e.g., supplements, fortified cereals, fruit juice, bread), our inverse associations for total iron and iron from supplements are not surprising. Research on dietary iron (Wurzelmann et al, 1996; Nelson, 2001; Kabat et al, 2007; Cross et al, 2010) or iron stores (Knekt et al, 1994; Herrinton et al, 1995; Wurzelmann et al, 1996; Cross et al, 2006) and colorectal cancer is inconclusive, and studies of prevalent or recurrent colorectal adenoma have also produced inconsistent results (Tseng et al, 1996, 1997, 2000; Chan et al, 2005; Ferrucci et al, 2009).

Combined nitrate and nitrite intake has been positively associated with colorectal adenoma (two-fold increased risk) (Ward et al, 2007), as has nitrate with colorectal cancer (16\% increased risk) (Cross et al, 2010). Yet, another study did not observe an association with nitrite and colorectal adenoma $(\mathrm{OR}=1.05,95 \% \mathrm{CI}=0.59-1.86)$ (Ferrucci et al, 2009), and both nitrite $(\mathrm{RR}=0.74,95 \% \mathrm{CI}=0.34-1.63)$ and nitrate $(\mathrm{RR}=1.04$, $95 \% \mathrm{CI}=0.54-2.02)$ were not related to colorectal cancer in another (Knekt et al, 1999). Further support for the NOC pathway in early colorectal neoplasia comes from our suggestive findings for processed meat and haeme iron. As processed meat, much of which is red meat, contains haeme iron, nitrate, and nitrite, these compounds may act synergistically during tumourigenesis. Haeme iron has been positively associated with endogenous $\mathrm{N}$-nitrosation (Cross et al, 2003), and nitrate and nitrite consumed from processed meat add to the pool of available nitrosating agents for reactions within the gastrointestinal tract. It is important to note that we did not evaluate other sources of nitrate and nitrite, and nitrate in particular is found in other foods and drinking water.

Our study had several strengths including a large number of cases and controls without distal colorectal adenoma confirmed with sigmoidoscopy screening. With detailed meat cooking and preparation information, we could evaluate multiple potentially carcinogenic meat-related components using quantitative databases. As adenoma is a largely asymptomatic condition, it should not have influenced diet in the year preceding the completion of the FFQ, thereby limiting the potential for reverse causation. Despite the relatively large sample size, our power to detect modest associations may still have been limited, especially for evaluating differences by advanced and/or multiple adenoma status and anatomical subsite. Our population was unique because we had information on colorectal cancer screening procedures for all participants; however, because those without adenoma only underwent sigmoidoscopy, we could not evaluate adenoma in the proximal colon. Although we had incident cases, as adenoma are largely asymptomatic and follow-up sigmoidoscopies occurred at defined intervals, we were not able to evaluate these relationships with time-to-event analyses. We were able to adjust for numerous potential confounders, but we did not have comprehensive information on previous history of lower gastrointestinal endoscopies. However, individuals reporting any lower gastrointestinal procedure (proctoscopy, sigmoidoscopy, barium enema, or colonoscopy) during the 3 years before study enrolment were excluded from the trial. Finally, the screening interval changed from 3 to 5 years after baseline during the course of the trial and could have impacted the type and number of adenomas detected, yet risks did not appear to vary when we stratified by screening interval.

In summary, we observed suggestive positive associations between red and processed meat, as well as grilled meat, well or very well-done meat, $\mathrm{PhIP}$, haeme iron, and nitrate/nitrite and distal colorectal adenoma. Grilled meat, well or very well-done meat, $\mathrm{PhIP}, \mathrm{B}[a] \mathrm{P}$, and mutagenic activity were all positively associated with rectal adenoma, highlighting potential aetiological differences in neoplasia throughout the colorectum. In contrast to the suggestive positive association we observed for haeme iron, we report inverse associations between total iron and iron from supplements and distal colorectal adenoma.

\section{ACKNOWLEDGEMENTS}

This research was supported (in part) by the Intramural Research Program of the National Institutes of Health, National Cancer Institute, by grant TU2 CA105666 from the National Cancer Institute, and by contracts from the Division of Cancer Prevention, National Cancer Institute, National Institutes of Health, Department of Health and Human Services. We thank Drs Christine Berg, Paul Pinsky, and Philip Prorok, Division of Cancer Prevention, National Cancer Institute, the Screening Center investigators and staff of the Prostate, Lung, Colorectal, and Ovarian (PLCO) Cancer Screening Trial, and $\mathrm{Mr}$ Tom Riley and staff, Information Management Services Inc.

\section{Conflict of interest}

The authors declare no conflict of interest. 


\section{REFERENCES}

Ames BN, McCann J, Yamasaki E (1975) Methods for detecting carcinogens and mutagens with the Salmonella/mammalian-microsome mutagenicity test. Mutat Res 31(6): $347-364$

Augustsson K, Skog K, Jagerstad M, Dickman PW, Steineck G (1999) Dietary heterocyclic amines and cancer of the colon, rectum, bladder, and kidney: a population-based study. Lancet 353(9154): $703-707$

Balder HF, Vogel J, Jansen MC, Weijenberg MP, van den Brandt PA, Westenbrink S, van der Meer R, Goldbohm RA (2006) Heme and chlorophyll intake and risk of colorectal cancer in the Netherlands cohort study. Cancer Epidemiol Biomarkers Prev 15(4): 717-725

Bogovski P, Bogovski S (1981) Animal species in which N-nitroso compounds induce cancer. Int J Cancer 27(4): 471-474

Burnett-Hartman AN, Newcomb PA, Mandelson MT, Adams SV, Wernli KJ, Shadman M, Wurscher MA, Makar KW (2011) Colorectal polyp type and the association with charred meat consumption, smoking, and microsomal epoxide hydrolase polymorphisms. Nutr Cancer 63(4): $583-592$

Butler LM, Sinha R, Millikan RC, Martin CF, Newman B, Gammon MD, Ammerman AS, Sandler RS (2003) Heterocyclic amines, meat intake, and association with colon cancer in a population-based study. Am J Epidemiol 157(5): $434-445$

Chan AT, Ma J, Tranah GJ, Giovannucci EL, Rifai N, Hunter DJ, Fuchs CS (2005) Hemochromatosis gene mutations, body iron stores, dietary iron, and risk of colorectal adenoma in women. J Natl Cancer Inst 97(12): 917-926

Cross AJ, Ferrucci LM, Risch A, Graubard BI, Ward MH, Park Y, Hollenbeck AR, Schatzkin A, Sinha R (2010) A large prospective study of meat consumption and colorectal cancer risk: an investigation of potential mechanisms underlying this association. Cancer Res 70(6): $2406-2414$

Cross AJ, Gunter MJ, Wood RJ, Pietinen P, Taylor PR, Virtamo J, Albanes D, Sinha R (2006) Iron and colorectal cancer risk in the alpha-tocopherol, beta-carotene cancer prevention study. Int J Cancer 118(12): 3147 - 3152

Cross AJ, Pollock JR, Bingham SA (2003) Haem, not protein or inorganic iron, is responsible for endogenous intestinal $\mathrm{N}$-nitrosation arising from red meat. Cancer Res 63(10): 2358-2360

Cross AJ, Sinha R, Wood RJ, Xue X, Huang WY, Yeager M, Hayes RB, Gunter MJ (2011) Iron homeostasis and distal colorectal adenoma risk in the prostate, lung, colorectal, and ovarian cancer screening trial. Cancer Prev Res (Phila) 4(9): 1465-1475

Culp SJ, Gaylor DW, Sheldon WG, Goldstein LS, Beland FA (1998) A comparison of the tumors induced by coal tar and benzo[a]pyrene in a 2-year bioassay. Carcinogenesis 19(1): 117-124

Ferrucci LM, Sinha R, Graubard BI, Mayne ST, Ma X, Schatzkin A, Schoenfeld PS, Cash BD, Flood A, Cross AJ (2009) Dietary meat intake in relation to colorectal adenoma in asymptomatic women. $\mathrm{Am} J$ Gastroenterol 104(5): 1231 - 1240

Fu Z, Shrubsole MJ, Smalley WE, Wu H, Chen Z, Yu S, Ness RM, Zheng W (2011) Association of meat intake and meat-derived mutagen exposure with the risk of colorectal polyps by histologic type. Cancer Prev Res (Phila) 4: $1686-1697$

Gerhardsson de Verdier M, Hagman U, Peters RK, Steineck G, Overvik E (1991) Meat, cooking methods and colorectal cancer: a case-referent study in Stockholm. Int J Cancer 49(4): 520-525

Giovannucci E, Stampfer MJ, Colditz G, Rimm EB, Willett WC (1992) Relationship of diet to risk of colorectal adenoma in men. J Natl Cancer Inst 84(2): 91 - 98

Glei M, Latunde-Dada GO, Klinder A, Becker TW, Hermann U, Voigt K, Pool-Zobel BL (2002) Iron-overload induces oxidative DNA damage in the human colon carcinoma cell line HT29 clone 19A. Mutat Res 519(1-2): $151-161$

Gunter MJ, Probst-Hensch NM, Cortessis VK, Kulldorff M, Haile RW, Sinha $\mathrm{R}$ (2005) Meat intake, cooking-related mutagens and risk of colorectal adenoma in a sigmoidoscopy-based case-control study. Carcinogenesis 26(3): $637-642$

Herrinton LJ, Friedman GD, Baer D, Selby JV (1995) Transferrin saturation and risk of cancer. Am J Epidemiol 142(7): 692-698

Ito N, Hasegawa R, Sano M, Tamano S, Esumi H, Takayama S, Sugimura T (1991) A new colon and mammary carcinogen in cooked food, 2-amino1-methyl-6-phenylimidazo[4,5-b]pyridine (PhIP). Carcinogenesis 12(8): $1503-1506$
Kabat GC, Miller AB, Jain M, Rohan TE (2007) A cohort study of dietary iron and heme iron intake and risk of colorectal cancer in women. $\mathrm{Br} J$ Cancer 97(1): 118-122

Kampman E, Slattery ML, Bigler J, Leppert M, Samowitz W, Caan BJ, Potter JD (1999) Meat consumption, genetic susceptibility, and colon cancer risk: a United States multicenter case-control study. Cancer Epidemiol Biomarkers Prev 8(1): $15-24$

Kesse E, Clavel-Chapelon F, Boutron-Ruault MC (2006) Dietary patterns and risk of colorectal tumors: a cohort of French women of the National Education System (E3N). Am J Epidemiol 164(11): 1085-1093

Knekt P, Jarvinen R, Dich J, Hakulinen T (1999) Risk of colorectal and other gastro-intestinal cancers after exposure to nitrate, nitrite and N-nitroso compounds: a follow-up study. Int I Cancer 80(6): 852-856

Knekt P, Reunanen A, Takkunen H, Aromaa A, Heliovaara M, Hakulinen T (1994) Body iron stores and risk of cancer. Int J Cancer 56(3): 379-382

Larsson SC, Adami HO, Giovannucci E, Wolk A (2005) Re: Heme iron, zinc, alcohol consumption, and risk of colon cancer. J Natl Cancer Inst 97(3): 232-233; author reply 233-234

Lee DH, Anderson KE, Harnack LJ, Folsom AR, Jacobs Jr DR (2004) Heme iron, zinc, alcohol consumption, and colon cancer: Iowa Women's Health Study. J Natl Cancer Inst 96(5): 403-407

Le Marchand L, Hankin JH, Pierce LM, Sinha R, Nerurkar PV, Franke AA, Wilkens LR, Kolonel LN, Donlon T, Seifried A, Custer LJ, Lum-Jones A, Chang W (2002) Well-done red meat, metabolic phenotypes and colorectal cancer in Hawaii. Mutat Res 506-507: 205-214

Lijinsky W (1992) Chemistry and Biology of N-Nitroso Compounds. Cambridge University Press: Cambridge

Martinez ME, Jacobs ET, Ashbeck EL, Sinha R, Lance P, Alberts DS, Thompson PA (2007) Meat intake, preparation methods, mutagens and colorectal adenoma recurrence. Carcinogenesis 28(9): 2019-2027

Mathew A, Sinha R, Burt R, Caan B, Paskett E, Iber F, Kikendall W, Lance P, Shike M, Weissfeld J, Schatzkin A, Lanza E (2004) Meat intake and the recurrence of colorectal adenomas. Eur J Cancer Prev 13(3): 159-164

Murtaugh MA, Ma KN, Sweeney C, Caan BJ, Slattery ML (2004) Meat consumption patterns and preparation, genetic variants of metabolic enzymes, and their association with rectal cancer in men and women. J Nutr 134(4): 776-784

Nelson RL (2001) Iron and colorectal cancer risk: human studies. Nutr Rev 59(5): $140-148$

Nowell S, Coles B, Sinha R, MacLeod S, Luke Ratnasinghe D, Stotts C, Kadlubar FF, Ambrosone CB, Lang NP (2002) Analysis of total meat intake and exposure to individual heterocyclic amines in a case-control study of colorectal cancer: contribution of metabolic variation to risk. Mutat Res 506-507: $175-185$

Ochiai M, Imai H, Sugimura T, Nagao M, Nakagama H (2002) Induction of intestinal tumors and lymphomas in $\mathrm{C} 57 \mathrm{BL} / 6 \mathrm{~N}$ mice by a food-borne carcinogen, 2-amino-1-methyl-6-phenylimidazo[4,5-b]pyridine. Jpn J Cancer Res 93(5): $478-483$

Ohgaki H, Takayama S, Sugimura T (1991) Carcinogenicities of heterocyclic amines in cooked food. Mutat Res 259(3-4): 399-410

Pierre F, Freeman A, Tache S, Van der Meer R, Corpet DE (2004) Beef meat and blood sausage promote the formation of azoxymethane-induced mucin-depleted foci and aberrant crypt foci in rat colons. J Nutr 134(10): $2711-2716$

Prorok PC, Andriole GL, Bresalier RS, Buys SS, Chia D, Crawford ED, Fogel R, Gelmann EP, Gilbert F, Hasson MA, Hayes RB, Johnson CC, Mandel JS, Oberman A, O'Brien B, Oken MM, Rafla S, Reding D, Rutt W, Weissfeld JL, Yokochi L, Gohagan JK (2000) Design of the Prostate, Lung, Colorectal and Ovarian (PLCO) Cancer Screening Trial. Control Clin Trials 21(6 Suppl): 273S - 309S

Robertson DJ, Sandler RS, Haile R, Tosteson TD, Greenberg ER, Grau M, Baron JA (2005) Fat, fiber, meat and the risk of colorectal adenomas. Am J Gastroenterol 100(12): 2789-2795

Rohrmann S, Hermann S, Linseisen J (2009) Heterocyclic aromatic amine intake increases colorectal adenoma risk: findings from a prospective European cohort study. Am J Clin Nutr 89(5): 1418-1424

Sesink AL, Termont DS, Kleibeuker JH, Van der Meer R (1999) Red meat and colon cancer: the cytotoxic and hyperproliferative effects of dietary heme. Cancer Res 59(22): 5704-5709

Sesink ALA, Termont DS, Kleibeuker JH, Van Der Meer R (2000) Red meat and colon cancer: dietary haeme, but not fat, has cytotoxic and hyperproliferative effects on rat colonic epithelium. Carcinogenesis 21(10): $1909-1915$ 
Shin A, Shrubsole MJ, Ness RM, Wu H, Sinha R, Smalley WE, Shyr Y, Zheng W (2007) Meat and meat-mutagen intake, doneness preference and the risk of colorectal polyps: the Tennessee Colorectal Polyp Study. Int $J$ Cancer 121(1): 136-142

Sinha R, Chow WH, Kulldorff M, Denobile J, Butler J, Garcia-Closas M, Weil R, Hoover RN, Rothman N (1999) Well-done, grilled red meat increases the risk of colorectal adenomas. Cancer Res 59(17): 4320-4324

Sinha R, Cross A, Curtin J, Zimmerman T, McNutt S, Risch A, Holden J (2005a) Development of a food frequency questionnaire module and databases for compounds in cooked and processed meats. Mol Nutr Food Res 49(7): 648-655

Sinha R, Knize MG, Salmon CP, Brown ED, Rhodes D, Felton JS, Levander OA, Rothman N (1998a) Heterocyclic amine content of pork products cooked by different methods and to varying degrees of doneness. Food Chem Toxicol 36(4): 289-297

Sinha R, Kulldorff M, Chow WH, Denobile J, Rothman N (2001) Dietary intake of heterocyclic amines, meat-derived mutagenic activity, and risk of colorectal adenomas. Cancer Epidemiol Biomarkers Prev 10(5): $559-562$

Sinha R, Kulldorff M, Gunter MJ, Strickland P, Rothman N (2005b) Dietary benzo[a]pyrene intake and risk of colorectal adenoma. Cancer Epidemiol Biomarkers Prev 14(8): 2030-2034

Sinha R, Peters U, Cross AJ, Kulldorff M, Weissfeld JL, Pinsky PF, Rothman N, Hayes RB (2005c) Meat, meat cooking methods and preservation, and risk for colorectal adenoma. Cancer Res 65(17): 8034-8041

Sinha R, Rothman N (1999) Role of well-done, grilled red meat, heterocyclic amines (HCAs) in the etiology of human cancer. Cancer Lett 143(2): $189-194$

Sinha R, Rothman N, Brown ED, Salmon CP, Knize MG, Swanson CA, Rossi SC, Mark SD, Levander OA, Felton JS (1995) High concentrations of the carcinogen 2-amino-1-methyl-6-phenylimidazo- [4,5-b]pyridine (PhIP) occur in chicken but are dependent on the cooking method. Cancer Res 55(20): 4516-4519

Sinha R, Rothman N, Salmon CP, Knize MG, Brown ED, Swanson CA, Rhodes D, Rossi S, Felton JS, Levander OA (1998b) Heterocyclic amine content in beef cooked by different methods to varying degrees of doneness and gravy made from meat drippings. Food Chem Toxico 36(4): $279-287$

Tappel A (2007) Heme of consumed red meat can act as a catalyst of oxidative damage and could initiate colon, breast and prostate cancers, heart disease and other diseases. Med Hypotheses 68(3): $562-564$

Tranah GJ, Giovannucci E, Ma J, Fuchs C, Hankinson SE, Hunter DJ (2004) Epoxide hydrolase polymorphisms, cigarette smoking and risk of colorectal adenoma in the Nurses' Health Study and the Health Professionals Follow-up Study. Carcinogenesis 25(7): 1211-1218

Tseng M, Greenberg ER, Sandler RS, Baron JA, Haile RW, Blumberg BS, McGlynn KA (2000) Serum ferritin concentration and recurrence of colorectal adenoma. Cancer Epidemiol Biomarkers Prev 9(6): 625-630

Tseng M, Murray SC, Kupper LL, Sandler RS (1996) Micronutrients and the risk of colorectal adenomas. Am J Epidemiol 144(11): 1005-1014

Tseng M, Sandler RS, Greenberg ER, Mandel JS, Haile RW, Baron JA (1997) Dietary iron and recurrence of colorectal adenomas. Cancer Epidemiol Biomarkers Prev 6(12): 1029-1032

Wang H, Yamamoto JF, Caberto C, Saltzman B, Decker R, Vogt TM, Yokochi L, Chanock S, Wilkens LR, Le Marchand L (2011) Genetic variation in the bioactivation pathway for polycyclic hydrocarbons and heterocyclic amines in relation to risk of colorectal neoplasia. Carcinogenesis 32(2): 203-209

Ward MH, Cross AJ, Divan H, Kulldorff M, Nowell-Kadlubar S, Kadlubar FF, Sinha R (2007) Processed meat intake, CYP2A6 activity and risk of colorectal adenoma. Carcinogenesis 28(6): 1210-1216

Willett WC (1998) Nutritional Epidemiology 2nd edn. Oxford University Press: New York

World Cancer Research Fund/American Institute for Cancer Research (2007) Food, Nutrition, Physical Activity, and the Prevention of Cancer: A Global Perspective. AICR: Washington, DC

Wu K, Giovannucci E, Byrne C, Platz EA, Fuchs C, Willett WC, Sinha R (2006) Meat mutagens and risk of distal colon adenoma in a cohort of U.S. men. Cancer Epidemiol Biomarkers Prev 15(6): 1120-1125

Wurzelmann JI, Silver A, Schreinemachers DM, Sandler RS, Everson RB (1996) Iron intake and the risk of colorectal cancer. Cancer Epidemiol Biomarkers Prev 5(7): $503-507$

This work is published under the standard license to publish agreement. After 12 months the work will become freely available and the license terms will switch to a Creative Commons Attribution-NonCommercial-Share Alike 3.0 Unported License. 